\title{
Adultos en situación de discapacidad
}

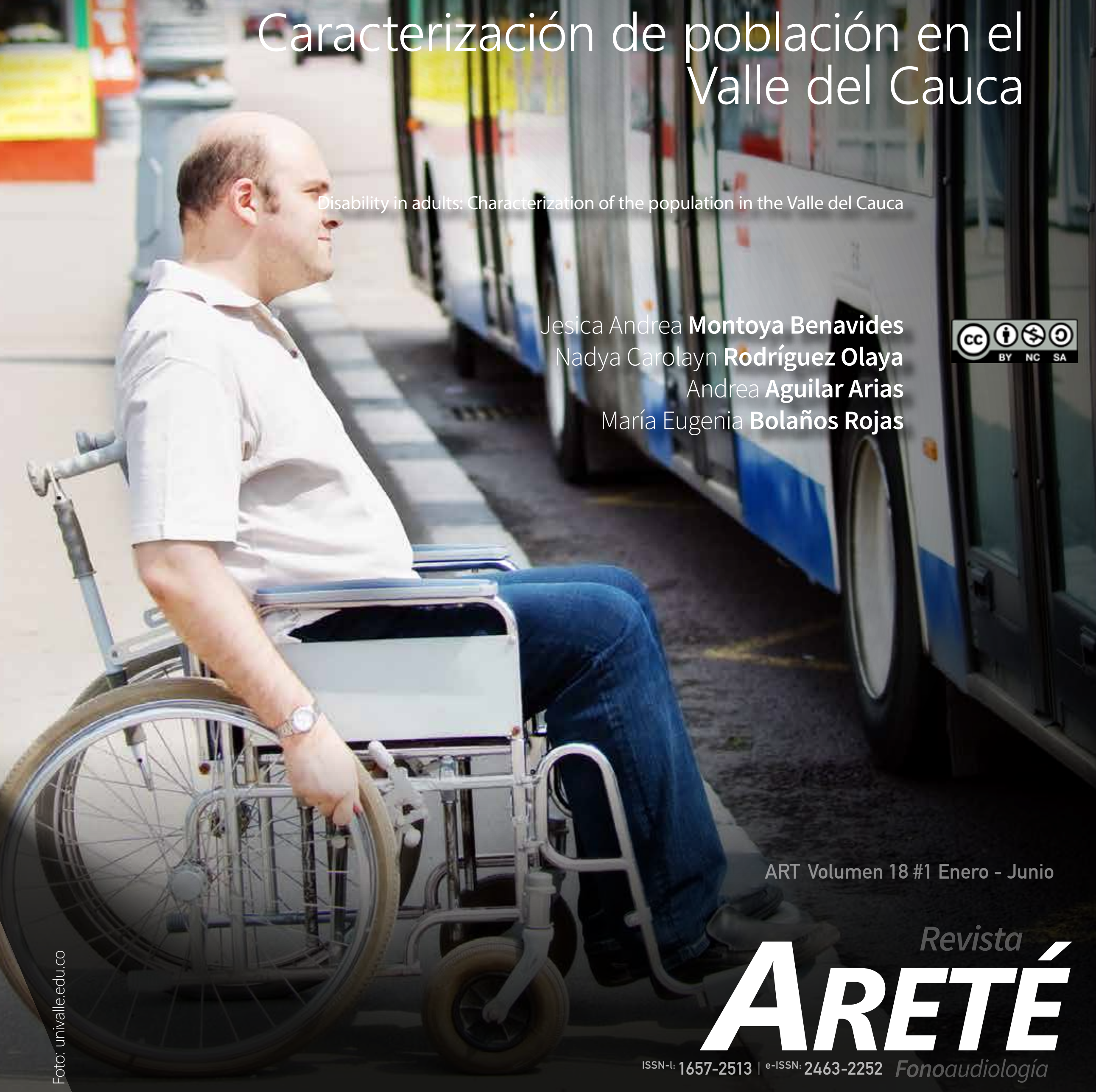




\section{ARETÉ \\ $1657-2513$ \\ 2463-2252 Fonoaudiología}

ID: 1657-2513.art.18104

Title: Disability in adults

Subtitle: Characterization of the population in the valle del cauca

Título: Adultos en situación de discapacidad

Subtítulo: Caracterización de población en el valle del cauca

Alt Title / Título alternativo:

[es]: Caracterización de población adulta con discapacidad en el valle del Cauca

Author (s) / Autor (es):

Montoya Benavides, Rodríguez Olaya, Aguilar Arias, \& Bolaños Rojas

Keywords / Palabras Clave:

[en]:*disabled persons; information systems; population; adults; colombia; record as topic

[es]: personas con discapacidad; sistemas de información; adultos; población; colombia; registros como asunto

Financiación / Funding:

Personas con discapacidad, Sistemas de

información, Adultos, Población, Colombia

Registros como Asunto

Submited: 2017-02-16

Acepted: 2017-09-26

\section{Andrea Aguilar Arias, MSc BHS}

AutorlD:**36958064600

ORCID:**0000-0002-2146-1741

Source | Filiacion:

Universidad del Valle

BIO:

Magister en Epidemiología Fonoaudiologa Docente - Investigador Universidad del Valle

City $\mid$ Ciudad:

Cali [Co]

e-mail:

andrea.aguilar@correounivalle.edu.co

Source | Filiacion:

\section{Resumen}

Este estudio responde a la necesidad de usar y divulgar la información del Registro de Localización y Caracterización de Personas con Discapacidad -RLCPD- en el Departamento del Valle del Cauca. El Objetivo fue caracterizar población con discapacidad mayor de 18 años 2009-

2014. Se utilizó como método el estudio descriptivo de población con discapacidad mayor de 18 años reportada en el RLCPD.

Incluyó variables sociodemográficas,

de salud, discapacidad y participación

social. Se presenta como resultados que durante el periodo analizado se reportaron

31.818 personas en los 42 municipios

del Departamento, 80\% residentes en cabeceras municipales. La distribución por sexo fue homogénea y el grupo con mayor concentración fue el de mayores de 60 años

$(n=11393) .67 \%$ reportó alguna deficiencia en estructuras o funciones; $49 \%$ limitaciones en la actividad y $87 \%$ reportó restricciones en la participación. Se evidenciaron barreras

en la residencia (44.2\%), en espacios públicos (49\%) y en establecimientos (60\%).

La mayor causa de discapacidad fue la enfermedad general (32\%), seguida de accidentes (12\%). El uso de ayudas técnicas fue reportado por el $64 \%$ de la población registrada. Finalmente, aunque se identificó que las restricciones en la participación tienen un alto porcentaje, el 58.4\% del total

de la población reporta que participa en actividades familiares

Jesica Andrea Montoya Benavides, [BHS]

Universidad del Valle

BIO:

Fonoaudióloga

City $\mid$ Ciudad:

Cali $[\mathrm{CO}]$

e-mail:

jessicaan0805@hotmail.com

\section{Abstract}

This study responds to the need to use and disseminate the information of the Register of Location and Characterization of Persons with Disabilities - RLCPD - in

the Department of Valle del Cauca. The objectives are to characterize the population with a disability over 18 years 2009-2014. It was used as method a descriptive study of the population with a disability over 18 years

of age reported in the RLCPD. It included sociodemographic variables, health, disability and social participation. it presents as results during the analyzed period, 31,818 people were reported in the 42 municipalities of the

Department, $80 \%$ of whom were residents of municipal head offices. The distribution by sex was homogeneous and the group with the highest concentration was those older

than 60 years $(n=11393) .67 \%$ reported some deficiency in structures or functions; $49 \%$ of activity limitations and $87 \%$ reported restrictions on participation. There were barriers in the residence (44.2\%), public spaces (49\%) and establishments (60\%). The major cause of disability was general illness (32\%), followed by accidents (12\%). The use

of technical aids was reported by $64 \%$ of the registered population. Finally, although

it was identified that the restrictions on participation have a high percentage, 58.4\%

of the total population reports that they participate in family activities.

\section{Nadya Carolayn Rodríguez Olaya, [BHS]}

Source | Filiacion:

Universidad del Valle

BIO:

Fonoaudióloga

City | Ciudad:

Cali [Co]

e-mail:

carolayn53@gmail.com

BIO:

BIO: Salud

City | Ciudad:

Cali $[\mathrm{CO}]$

e-mail:

mbolanos@valledelcauca.gov.co dad:

Citar como:

Montoya Benavides, J. A., Rodríguez Olaya, N. C., Aguilar Arias, A., \& Bolaños Rojas, M. E. (2018). Adultos en situación de discapacidad: Caracterización de población en el valle del cauca. Revista Areté, Fonoaudiología, 18 (1), 40-47]. Obtenido de: https://revistas.iberoamericana.edu.co/index.php/ripsicologia/ article/view/1164 


\title{
Adultos en situación de discapacidad Caracterización de población en el valle del Cauca
}

Disability in adults: Characterization of the population in the Valle del Cauca

\author{
Jesica Andrea Montoya Benavides \\ Nadya Carolayn Rodríguez Olaya \\ Andrea Aguilar Arias \\ María Eugenia Bolaños Rojas
}

\section{Introducción}

El estudio presentado a continuación se realizó en alianza con la Secretaria Departamental de Salud del Valle del Cauca y el Grupo de Investigación al cual pertenece el Programa Académico de Fonoaudiología de la Universidad del Valle, su propósito es aportar información que contribuya a la identificación de necesidades, atención, inclusión social y de equiparación de oportunidades de las Personas con Discapacidad - (PCD en adelante) del departamento, mediante su caracterización general.

La discapacidad entendida como un evento multicausal que va desde causas pre y peri natales, procesos naturales de envejecimiento, hasta causas sociales, afecta no solo al individuo sino también a su familia y al entorno más cercano, por lo tanto, el panorama requiere de una intervención que va más allá de los servicios de salud, pues se convierte en un motivo de preocupación de los entes gubernamentales mundiales debido a que su prevalencia aumenta cada vez más.

Según la Organización Mundial de la Salud - OMS (2011), se estimó que para el año 2010 habían más de 100 millones de personas que vivían con algún tipo de discapacidad; ahora bien, este indicador prende las alarmas acerca de la necesidad de realizar análisis detallados con información confiable y actualizada de los indicadores de salud; estos análisis deben permitir caracterizar, medir y explicar el contexto de la salud y la discapacidad de la población.

En Colombia datos obtenidos por el Departamento Administrativo Nacional de Estadística-DANE (2005) arrojaron que el total de personas con por lo menos una limitación fue de 2'632,255 y específicamente para el Valle del Cauca se identificaron $\mathbf{2 6 7 , 3 4 8}$ personas con limitaciones permanentes; posteriormente en el año 2008 el DANE presentó los resultados preliminares de la implementación del Registro para la Localización y Caracterización de las Personas con Discapacidad - RLCPD (DANE, 2008); en el que para el Valle del Cauca se identificaron 59,472 $\boldsymbol{P C D}$ registradas y de acuerdo al análisis realizado para los diferentes grupos de edad, las personas mayores de 60 años son quienes presentan un dato más relevante.

Para obtener este tipo de información se deben realizar mediciones en discapacidad, por tanto la OMS en el año 2001, desarrolló la Clasificación Internacional del Funcionamiento, de la Discapacidad y de la Salud - CIF la cual visibiliza la discapacidad como un resultado entre la interacción del individuo y los factores contextuales, además gracias a su lenguaje estandarizado y unificado posibilita la comunicación sobre salud y la atención sanitaria (WHO/OMS, 2001).

En este año además, se realizó un estudio de prevalencia de discapacidad en el departamento del Valle 
del Cauca, en alianza entre la Escuela de Rehabilitación Humana de la Universidad del Valle y la Secretaría Departamental de Salud (SDS, 2011); este estudio permite obtener cifras que datan acerca de la frecuencia de la discapacidad de la población, además se convierte en un precedente en la implementación de una herramienta estadística basada en la propuesta de la $\boldsymbol{C I} \boldsymbol{F}$, esta herramienta se consolida como un antecedente en la creación de un formato de registro de $\boldsymbol{P C D}$

Además, desde el año 2003 en el Valle del Cauca se cuenta con el Sistema Integral de Información de la Protección Social - SISPRO proporcionando a los actores del sistema de salud y protección social el acceso a la información de manera confiable y oportuna. El proceso de registro de las $\boldsymbol{P C D}$ se lleva a cabo de la siguiente manera: se identifican las entidades receptoras de la población con discapacidad del departamento, se establecen y capacitan como unidades generadoras de datos - UGD allí se encuentra personal para diligenciar el formato del $\boldsymbol{R} \boldsymbol{L C P D}$ el cual es un formulario electrónico en el que se registra la información sobre la salud y participación de la población con discapacidad, finalmente, esta información queda condensada en el SISPRO para ser utilizada.

Las preguntas del $\boldsymbol{R}$ LCPD fueron basadas en la $\boldsymbol{C I F}$ y su importancia radica en que contiene información veraz y oportuna para la toma de decisiones, así como la orientación de programas y proyectos.

Se planteó como hipótesis en la investigación que los datos recogidos en el $\boldsymbol{R} \boldsymbol{L C P D}$ aportan información pertinente y necesaria para proyectar acciones de atención a la población con discapacidad en el Departamento del Valle del Cauca.

\section{Objetivos}

Como objetivo general se propuso: Caracterizar la población con discapacidad mayor de 18 años reportada en el Registro de Localización y Caracterización de Personas con Discapacidad RLCPD en el Valle del Cauca en el periodo 2009 - 2014.

\section{Objetivos específicos}

$\rightarrow$ Describir aspectos sociodemográficos (año de diligenciamiento, edad, sexo, tipo de área y municipio) de la población objeto de estudio.

$\rightarrow$ Describir características referidas a la discapacidad (deficiencia en estructura/ función, limitaciones en la actividad, barreras, consecuencia de la discapacidad) y la salud (uso de ayudas) en la población de estudio.

$\rightarrow$ Identificar aspectos relacionados con la participación de las personas con discapacidad (participación y restricciones en la participación).

\section{Método}

Se llevó a cabo un estudio descriptivo a través del análisis de información existente en una base de datos con registro de datos rutinario, la fuente de información fue secundaria, específicamente los datos registrados en la base de datos del SISPRO.
Para la inclusión de los datos reportados en la base de datos

Personas con Discapacidad se tuvo en cuenta:

$\rightarrow$ El $\boldsymbol{R} \boldsymbol{L C P D}$ debía tener fecha de diligenciamiento entre el año 2009 y 2014.

$\rightarrow$ Registros diligenciados en año calendario finalizado.

$\rightarrow$ Registros que correspondieran a personas reportadas como mayores de 18 años de edad.

$\rightarrow$ Registros de $\boldsymbol{P C D}$ que, aunque hubieran diligenciado el $\boldsymbol{R} \boldsymbol{L C P D}$ en otro departamento y municipio, reportaran como residencia un municipio del departamento del Valle del Cauca.

$\rightarrow$ Registros completos según la variable de la base de datos "estado".

Se hizo uso del programa de hojas de cálculo Microsoft Excel para filtrar la información que haría parte del estudio, posteriormente se adicionaron variables de interés propias y finalmente se realizó el análisis de los datos, a través del paquete estadístico Stata versión 12.0 free online.

\section{Resultados}

Se presenta a continuación el análisis estadístico de los 31,818 registros ${ }^{1}$ de PCD mayor de 18 años, reportados en el RLCPD durante el periodo 2009-2014 en el Departamento del Valle del Cauca. Para cada una de las variables se obtuvieron las distribuciones de frecuencia en valores absolutos (n) y porcentuales (\%).

\section{Características sociodemográficas}

Para el año 2013 se reportó el porcentaje más alto de diligenciamiento del RLCPD (28.5\%), mientras que para el año 2011 se encontraron tan solo 10 registros diligenciados, lo que arroja un porcentaje del 0.03\%; durante este año (2011), los únicos municipios que tienen reporte son: Buenaventura $(n=4)$; Caicedonia $(n=1)$, Jamundí $(n=2)$, Palmira $(n=2)$ y Yumbo $(n=1)$.

Las diferencias en el número de registros en el periodo analizado son notorias entre los municipios, encontrando en algunos reportes menores de 5 datos y en otros, cifras por encima de los $\mathbf{1 , 0 0 0}$ registros (Ver Tabla 1).

Tabla 1 Municipio de residencia de las personas con discapacidad

\begin{tabular}{cccccccc}
$\#$ & Municipio & $\mathrm{n}$ & $\%$ & $\#$ & Municipio & $\mathrm{n}$ & $\%$ \\
\hline 1 & Cali & 9.406 & 29.56 & 22 & Jamundí & 556 & 1.75 \\
2 & Alcalá & 3 & 0.01 & 23 & La Cumbre & 67 & 0.21 \\
3 & Andalucía & 1 & 0.00 & 24 & La Unión & 778 & 2.45 \\
4 & Ansermanuevo & 2 & 0.01 & 25 & La Victoria & 4 & 0.01 \\
5 & Argelia & 2 & 0.01 & 26 & Obando & 10 & 0.03 \\
6 & Bolívar & 3 & 0.01 & 27 & Palmira & 5.817 & 18.28 \\
7 & Buenaventura & 1.176 & 3.70 & 28 & Pradera & 2 & 0.01 \\
8 & Bugalagrande & 3 & 0.01 & 29 & Restrepo & 0 & 0 \\
9 & Caicedonia & 496 & 1.56 & 30 & Riofrío & 204 & 0.64 \\
10 & Calima & 126 & 0.40 & 31 & Roldanillo & 4 & 0.01
\end{tabular}

Como se indicó en el apartado de metodología se incluyeron en este estudio solamente los registros que tenían en la variable estado la opción: completo 
Adultos en situación de discapacidad

Caracterización de población en el valle del cauca

$\begin{array}{lccccccc}11 & \text { Candelaria } & 914 & 2.87 & 32 & \text { San Pedro } & 0 & 0 \\ 12 & \text { Cartago } & 2.221 & 6.98 & 33 & \text { Sevilla } & 166 & 0.52 \\ 13 & \text { Dagua } & 816 & 2.56 & 34 & \text { Toro } & 3 & 0.01 \\ 14 & \text { El Águila } & 5 & 0.02 & 35 & \text { Trujillo } & 753 & 2.37 \\ 15 & \text { El Cairo } & 3 & 0.01 & 36 & \text { Tuluá } & 4.901 & 15.40 \\ 16 & \text { El Cerrito } & 1.035 & 3.25 & 37 & \text { Ulloa } & 1 & 0.00 \\ 17 & \text { El Dovio } & 1 & 0.00 & 38 & \text { Versalles } & 4 & 0.01 \\ 18 & \text { Florida } & 99 & 0.31 & 39 & \text { Vijes } & 203 & 0.64 \\ 19 & \text { Ginebra } & 0 & 0 & 40 & \text { Yotoco } & 29 & 0.09 \\ 20 & \text { Guacarí } & 5 & 0.02 & 41 & \text { Yumbo } & 1.463 & 4.60 \\ 21 & \text { Guadalajara de } & 535 & 1.68 & 42 & \text { Zarzal } & 1 & 0.00\end{array}$

Fuente: + Caracterización de la población con discapacidad en el Valle del Cauca. Base de datos de personas con discapacidad. Secretaria Departamental de Salud; Cali, 2015.

Cuando se analizó la distribución de los datos según el área de residencia de las $\boldsymbol{P C D}$ se encontró que el $\mathbf{8 0} \%$ de la población reside en las cabeceras municipales y el $\mathbf{2 0} \%$ restante se ubica en la zona rural.

El municipio que reportó un mayor número de registros para el periodo analizado fue Cali $\mathbf{2 9 . 5 \%}$, seguido por Palmira $\mathbf{1 8 . 2 \%}$, Tuluá $\mathbf{1 5 . 4 \%}$, Cartago $6.9 \%$, Yumbo $4.6 \%$ y Buenaventura $3.7 \%$, mientras que, en los municipios de Ginebra, Restrepo y San Pedro, no se encontró registrada ninguna $\mathbf{P C D}$ en el periodo de estudio.

Por otra parte, al realizar un análisis sobre la cantidad de datos reportados por año y municipio, se identificó que para el municipio de Cali durante el año 2009 se reportó el más alto diligenciamiento de registros con $\mathbf{5 , 2 6 0}$, no obstante, para la ciudad de Palmira se evidenció que, durante los años 2009, 2010 y 2011 el registro de la población con discapacidad fue mínimo, sin embargo, para el año 2012 hubo un incremento considerable, registrando así $\mathbf{5 , 7 4 4}$ datos.

Con respecto a la ubicación de la vivienda según el tipo de área, se encontró que el $\mathbf{8 0 . 2 \%}$ de la población registrada se localiza en las cabeceras municipales y el $\mathbf{1 3} \%$ restante refiere vivir en centro poblado - zona rural.

La distribución por sexo fue homogénea encontrando que $\mathbf{5 0 . 9 \%}$ de las personas registradas fueron hombres y $\mathbf{4 9 \%}$ mujeres; además, se pudo apreciar la distribución por grupos de edad de la población con discapacidad registrada, presentando por lo general un ligero aumento de forma progresiva a partir del grupo de 18 a 22 años, siendo así el mayor porcentaje en el grupo de 63 años en adelante con un $\mathbf{3 5 . 8 \%}$; para facilitar el análisis la información se organizó por quinquenios.

\section{Características de la}

Los datos reportados se clasificaron de acuerdo a la CIF en deficiencias, limitaciones en la actividad, restricciones en la participación y barreras para la participación.

\section{Deficiencias en estructura/función y} alteraciones

\section{Limitaciones en la actividad y dificultades}

Con respecto al reporte que hizo la población registrada sobre dificultades para realizar algunas actividades cotidianas, el $48.6 \%$ refirió limitaciones en la actividad, la mayoría para movilizarse 22.6\%; otras limitaciones importantes se refirieron a las dificultades para hablar y comunicarse correspondiente al $17.6 \%$ y para oír $14.1 \%$.

\section{Barreras}

Los datos presentados en la figura 2 muestran la presencia de barreras en algunos de los contextos en los cuales se desenvuelve generalmente el ser humano; en la casa/ residencia hubo un porcentaje de $37.7 \%$ perteneciente específicamente a las escaleras (ver tabla 2), este dato es correspondiente al tipo de alteración con mayor frecuencia, el movimiento corporal, en este sentido, dicha alteración dificulta el desplazamiento de las personas a través de estructuras físicas como las escaleras.

En los espacios públicos la calle fue reportada con mayor presencia de barreras, seguido de los andenes y los vehículos de transporte público, $\mathbf{3 7 . 5 \%}, \mathbf{2 9 . 2 \%}$ y $\mathbf{2 9} \%$ respectivamente; y en menor medida las barreras en los centros educativos, no obstante, hay que tener en cuenta que la mayoría de la población registrada corresponde a personas mayores de 63 años y es en esta edad donde disminuye la asistencia según el reporte del DANE en el 2008 "a partir de los 14 años la asistencia escolar disminuye en forma importante y conforme aumenta la edad". (DANE, 2008)

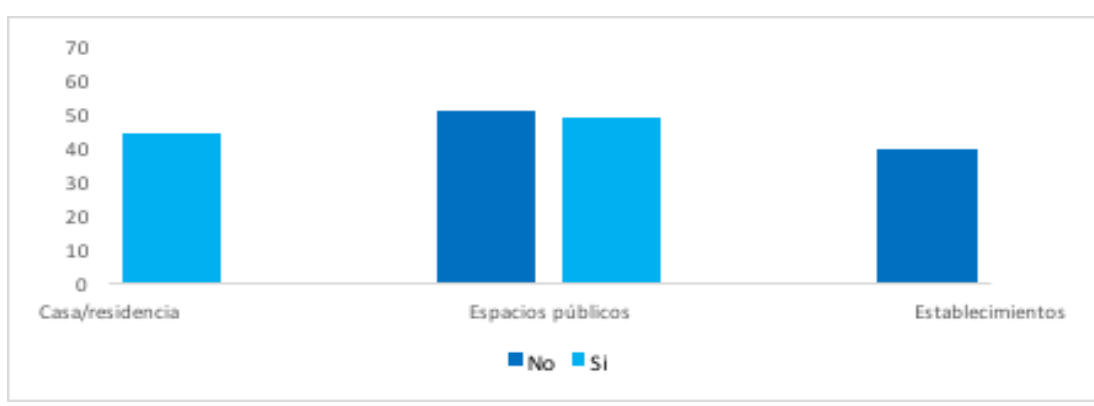

Grafico 2. Barreras

Fuente: Elaboración propia. Caracterización de la población con discapacidad en el Valle del Cauca; Cali, 2015. 
Tabla 2 Barreras

$\begin{array}{ccc}\begin{array}{c}\text { ¿En qué lugares de su vivienda o entorno físico, encuentra } \\ \text { barreras que le impidan desarrollar sus actividades diarias } \\ \text { con mayor autonomía? }\end{array} & n & \% \\ \text { Dormitorio } & 2.388 & 7.51 \\ \text { Sala } & 2.311 & 7.26 \\ \text { Baño } & 4.278 & 13.45 \\ \text { Escaleras } & 12.016 & 37.76 \\ \text { Pasillos } & 5.035 & 15.82 \\ \text { Andenes } & 9.31 & 29.26 \\ \text { Calle } & 11.944 & 37.54 \\ \text { Parques, plazas, estadios, teatros } & 6.166 & 19.38 \\ \text { Paraderos, terminales de transporte. } & 6.372 & 20.03 \\ \text { Vehículos de transporte público } & 9.247 & 29.06 \\ \text { Centros educativos } & 3.218 & 10.11 \\ \text { Lugares de trabajo } & 4.074 & 12.80 \\ \text { Centros de salud, hospitales } & 4.101 & 12.89 \\ \text { Centros comerciales, tiendas, plazas de mercado. } & 4.435 & 13.94\end{array}$

Fuente: *** Caracterización de la población con discapacidad en el Valle del Cauca. Base de datos de personas con discapacidad. Secretaria Departamental de Salud; (MinSalud, 2015)

\section{Causa de la discapacidad y uso de ayudas}

Con respecto al origen de la discapacidad, el mayor porcentaje identifica como causa de la discapacidad a la enfermedad general con un 32.1\%, seguido del accidente 12.8\%; las dificultades en la prestación de servicios de salud también hicieron parte de las causas, no obstante, lo fue en menor proporción 0.9\%; al identificar el uso de ayudas, el 35.7\% correspondiente a 11,375 personas registradas, refirieron el uso de medicamentos.

\section{Participación de las personas con discapacidad}

\section{Restricciones en la participación}

En este estudio el porcentaje referente a las restricciones en la participación fue de $87.3 \%$, los datos mostraron que las personas atribuyen como causas de su no participación al impedimento en el desarrollo de las actividades, generado por los familiares, vecinos y amigos $6.4 \%$, 3.1\% y $3 \%$ respectivamente.

\section{Participación en actividades}

El $76.6 \%$ de la población registrada refirió participar en diferentes actividades, la mayor participación se encuentra para las actividades familiares con un 58.4\%, y una menor participación de la población se encuentra reportada para las actividades ciudadanas 3.1\%. (Tabla 3)
Tabla 3 Participación en Actividades

$\begin{array}{ccc}\text { Participa en actividades } & \mathrm{n} & \% \\ \text { Familiares } & 18.583 & 58.40 \\ \text { Con la comunidad } & 7.412 & 23.29 \\ \text { Religiosas } & 13.397 & 42.11 \\ \text { Productivas } & 1.47 & 4.62 \\ \text { Deportivas } & 2.931 & 9.21 \\ \text { Culturales } & 1.681 & 5.28 \\ \text { De educación } & 432 & 1.36 \\ \text { Ciudadanas } & 1.006 & 3.16 \\ \text { Otras actividades } & 1.308 & 4.11 \\ \text { Fuente: }{ }^{* * * *} \text { Caracterización de la población con discapacidad en el Valle } \\ \text { del Cauca. Base de datos de personas con discapacidad. Secretaria } \\ \\ \text { Departamental de Salud; (MinSalud, 2015) }\end{array}$

A partir de la información obtenida de la base de datos analizada, se logró elaborar un perfil descriptivo de la población con discapacidad mayor de 18 años registrada en los RLCPD con lugar de residencia en el Departamento del Valle del Cauca durante el periodo 2009-2014, con este análisis se logra reconocer e identificar que existen diversos factores que influyen en las necesidades de atención, el desempeño autónomo de esta población y por ende en su calidad de vida.

En los 6 años estudiados, a partir del análisis sobre la cantidad de datos reportados por año y municipio, se evidenciaron variaciones importantes entre la cantidad de reportados en los diferentes años, lo que dificulta llevar a cabo comparaciones con el comportamiento de los datos por municipio, no obstante, se puede presumir que dichas variaciones se deben a la capacidad instalada para recoger el dato de las entidades UGD en los diferentes municipios.

Según el reporte del Plan Decenal de Salud Pública - PDSP 2012-2021 (2015), dentro del proceso de transformación de la población colombiana se destaca su distribución en el territorio nacional, identificándose una tendencia a ocupar territorios urbanos en lugar de los rurales, además según el DANE, Colombia pasó de tener alrededor del $\mathbf{3 0} \mathbf{\%}$ de la población residente en las zonas urbanas -cabeceras municipales- a tener las tres cuartas partes de su población (75\%) establecidas en estas zonas; el análisis de la información contenida en esta investigación, permitió poner de manifiesto esta relación, puesto que de los $\mathbf{3 1 , 8 1 8}$ registros analizados, $\mathbf{9 , 4 0 6}$ es decir el $\mathbf{2 9 . 5 \%}$ del total de la población incluida en el estudio identificó que la ciudad de Cali, es la que presenta mayor concentración de esta población; además, con relación a lo anterior y según reporte del $\boldsymbol{P D S P}$, se puede observar que Cali se encuentra entre las ciudades que concentran el $\mathbf{4 0 . 6 \%}$ del total de la población nacional, siendo un punto de partida para que desde las distintas instancias gubernamentales se desarrollen planes de acción para la prevención de la discapacidad con la mejora de las acciones de la Política Nacional y Territorial de Discapacidad.

Por su parte en el 2011 en el Informe Mundial sobre la Discapacidad, (WHO/OMS, 2011) se estableció que el número de PCD ha incrementado debido al envejecimiento y al incremento global de los problemas crónicos de la salud, afectando a las poblaciones más vulnerables, lo cual podría llevar a suponer que la población adulta mayor se encuentra dentro de este criterio, teniendo en 


\section{Caracterización de población en el valle del cauca}

cuenta que se identificó que es el grupo poblacional con mayor porcentaje de diligenciamiento del registro, esto constituye un evidente predominio de discapacidad en la edad avanzada; de esta manera, se puede presumir que la enfermedad general es la razón principal para que el grupo de edad mayor a los 63 años sea quien evidencie mayor porcentaje (35.8\%), además la causa principal de la discapacidad según este estudio es la enfermedad general con el 32.1\%; a partir de lo anterior se evidencia una constante debido a que en el informe del 2008 (DANE, 2008) elaborado por el DANE se estableció que en promedio las personas menores de 60 años presentaban prevalencia del $\mathbf{1 0 . 3} \%$ por cada mil personas, mientras que en los mayores de 60 años el dato aumentaba al $\mathbf{5 4 . 6 \%}$. Los datos anteriores presentan una relación entre la enfermedad general y el uso de medicamentos, en coherencia son estos datos los que presentan mayor porcentaje, y se relacionan teniendo en cuenta que cualquier enfermedad genera el uso de medicamentos como parte del tratamiento.

Para comprender y analizar la discapacidad desde una perspectiva biopsicosocial se establece una mirada a partir de los fundamentos de la CIF, la cual define desde una visión integradora la presencia de barreras como interventores en la presencia de discapacidad; identificando además deficiencias, limitaciones en la actividad y restricciones en la participación de las personas con discapacidad.

Las deficiencias con mayor frecuencia según los resultados de la investigación corresponden al movimiento corporal con $\mathbf{5 0 . 7 \%}$, mientras que a nivel auditivo y de voz se reporta menos del $\mathbf{4 0 \%}$; en coherencia, en el estudio de Prevalencia del 2001 se estableció el predominio de esta alteración, al igual que en el Informe del DANE en el 2008, (DANE, 2008) donde se identificó que el $\mathbf{5 3 . 8 \%}$ presentaba alteración en el movimiento del cuerpo mientras que la afección de la voz y el sistema auditivo era de $\mathbf{5 2 . 2 \%}$, siendo la diferencia mínima $\mathbf{1 . 6 \%}$, no obstante, a partir de este estudio se establece la disminución de alteraciones en la voz y la audición con respecto al mantenimiento del porcentaje en movimiento corporal, la diferencia radica en $\mathbf{1 4 . 4 \%}$.

Con respecto al reporte que se hace de la población registrada, se evidencia la dificultad para realizar algunas actividades cotidianas, el $\mathbf{4 8 . 6 \%}$ reporta limitaciones en la actividad, la mayoría para movilizarse $\mathbf{2 2 . 6 8 \%}$ lo que se concierne a la dificultad existente en algunas funciones o alteración en alguna estructura (deficiencia); sin embargo, las dificultades para hablar y comunicarse, que de manera implícita implica las estructuras y función de la voz, se encuentran con un porcentaje del $\mathbf{1 7 . 6 \%}$. Los resultados arrojados en dificultad para relacionarse $\mathbf{1 0 . 1 1 \% ,}$ se encuentran correlacionados con los porcentajes que definen a las actividades como oír, hablar y comunicarse, debido que, al presentarse una dificultad a nivel comunicativo, las relaciones interpersonales se ven afectadas, específicamente disminuidas. Los datos anteriores se relacionan igualmente con las barreras que predominan, siendo estas en lugares o estructuras físicas que implican una mayor demanda física, por ejemplo, el porcentaje de barrera perteneciente a las escaleras, relacionada con la alteración de mayor predominio, el movimiento corporal.

Los resultados proporcionados anteriormente van de la mano con los planteamientos de la $\mathbf{C I F}$ y las restricciones en la participación, las cuales se derivan del impedimento para el desarrollo de actividades. Por su parte, la participación en actividades con menor predominio son las educativas (1.3\%), este porcentaje pertenece principalmente al grupo poblacional mayor a 60 años, teniendo en cuenta que el DANE en su informe del 2008 refiere que "a partir de los 14 años la asistencia escolar disminuye en forma importante y conforme aumenta la edad" (DANE, 2008, pág. S.P.)

Ahora bien, la caracterización de la población con discapacidad del departamento del Valle del Cauca fue obtenida en este estudio a partir de la información contenida en el $\boldsymbol{R} \boldsymbol{L C} \boldsymbol{C P D}$, la importancia del Registro radica en conocer el número de personas que presentan alguna discapacidad en el Municipio, Departamento, Distrito y en general, en todo el país, para disponer de información veraz y oportuna para la toma de decisiones, así como para la orientación de programas y proyectos a nivel municipal, departamental / distrital y nacional; en coherencia se instaura la importancia de su implementación debido a que con este se logra obtener información que permite recopilar información estadística adecuada que posibilite la formulación y aplicación de las políticas dirigidas a la población con discapacidad, de acuerdo a lo establecido en la Ley 1346 (2009) en su artículo número 31; por tal razón, se define la necesidad de mantener actualizado el dato y de realizar un análisis constante de la información.

\section{Conclusiones}

La información que recoge el $\boldsymbol{R}$ LCPD que tiene como base la Clasificación Internacional del Funcionamiento, de la Discapacidad y de la Salud - CIF logra identificar las barreras físicas y actitudinales que refiere la población con discapacidad mayor de 18 años en el Departamento del Valle del Cauca, evidenciando que más del $\mathbf{5 0 \%}$ de las personas encuestadas reporta barreras en espacios públicos sobre los cuales se pueden proyectar acciones en concordancia con la Ley 1346 (2009) que plantea la Accesibilidad para "... personas con discapacidad en igualdad de condiciones con las demás, al entorno físico, el transporte, la información y las comunicaciones..., y a otros servicios $e$ instalaciones abiertos al público o de uso público, tanto en zonas urbanas como rurales..."

Desarrollar investigaciones con información confiable y actualizada es necesario para que, en los niveles de políticas y programas, los líderes y encargados de la toma de decisiones logren focalizar propuestas que respondan a las verdaderas necesidades de la población con discapacidad mayor de 18 años que se vive en el Departamento.

A través de las investigaciones que se desarrollen en alianzas entre la Academia, en este caso la Universidad del Valle y el Gobierno, en este caso la Secretaría Departamental de Salud, se logra disponer de datos actualizados sobre la población con discapacidad residente en el departamento, lo cual favorece la planificación, ejecución y seguimiento de acciones por parte de los tomadores de decisiones en el tema de la atención a la discapacidad.

La mayor frecuencia de discapacidad en los reportes se evidenció en población adulta mayor de 60 años, lo cual corresponde con la causa de la discapacidad más reportada que es la enfermedad general y con las ayudas técnicas más requeridas como bastón, gafas y/o audífono. 


\section{Agradecimientos}

A la Secretaría Departamental de Salud por facilitar la base de datos para este estudio.

\section{Trabajos citados}

1. Congreso de la República de Colombia. (28 de 05 de 2018). Alcaldía de Bogotá. Obtenido de Ley 1346 de 2009: http://www.alcaldiabogota. gov.co/sisjur/normas/Norma1.jsp?i=37150

2. Departamento Administrativo Nacional de Estadística. (2005). Censo General 2005. Colombia.

3. Departamento Administrativo Nacional de Estadística. (2008). Resultados preliminares de la implementación del registro para la localización y caracterización de las personas con discapacidad Departamento Valle del Cauca. Colombia.

4. Gobernación del Valle del Cauca, Secretaría Departamental de Salud. (2011). Análisis de situación en salud - ASIS. Colombia.

5. Gómez, N., Otoya, M., Quintana, Q., Tenorio, L., Vergara, C., \& Zapata, M. (2001). Prevalencia de Discapacidad en el Departamento del Valle del Cauca.

6. Ministerio de Salud y protección Social - MINSALUD. (5 de Febrero de 2015). Sistema Nacional de Discapacidad - SND. Obtenido de https:// www.minsalud.gov.co/proteccionsocial/Paginas/Sistema\%20Nacional\%20 de\%20Discapacidad.aspx.

7. Ministerio de Salud y Protección Social- República de Colombia. (13 de Mayo de 2015). Plan Decenal de Salud Pública 2012-2021. Obtenido de http://www.minsalud.gov.co/Documentos\%20y\%20Publicaciones/ Plan\%20Decenal\%20-\%20Documento\%20en\%20consulta\%20para\%20 aprobaci\%C3\%B3n.pdf

8. Organización Mundial de la Salud. (2001). Clasificación internacional del funcionamiento, la discapacidad y la salud CIF. OMS/ OPS.

9. Organización Mundial de la Salud. (2011). Informe mundial sobre discapacidad. OMS/ Banco Mundial. 